BI-TP 95/21

FSU-SCRI-95-53

May 1995

\title{
The Gluon Propagator at High Temperature
}

\author{
U.M. Heller ${ }^{1}$, F. Karsch ${ }^{2}$, J. Rank ${ }^{2}$ \\ ${ }^{1}$ SCRI, Florida State University, Tallahassee, FL 32306-4052, USA \\ ${ }^{2}$ Fakultät für Physik, Universität Bielefeld, P.O. Box 100131, D-33501 Bielefeld, \\ Germany
}

\begin{abstract}
We study the gluon propagator in Landau gauge in the deconfined phase of $S U(2)$ gauge theory. From the long-distance behaviour of correlation functions of temporal and spatial components of the gauge fields we extract electric $\left(m_{e}\right)$ and magnetic $\left(m_{m}\right)$ screening masses. For temperatures larger than twice $T_{c}$ we find no additional temperature dependence in $m_{e}(T) / T$, while $m_{m}(T) / T$ drops with increasing temperature. The decrease is consistent with the expected behaviour, $m_{m}(T) \sim g^{2}(T) T$. We find $m_{e}(T)=2.484(52) T$ and $m_{m}(T)=0.466(15) g^{2}(T) T$.
\end{abstract}


A basic non-perturbative feature of the high temperature plasma phase of QCD is the occurrence of electric and magnetic screening masses for the gluon. They play an important role in controlling the infrared behaviour of QCD [1]. While the electric screening mass can be calculated in leading order perturbation theory and is found to be $O(g T)$, little is known about the magnitude of the magnetic screening mass, which is expected to be $O\left(g^{2} T\right)$ [1]. However, also other functional dependences have been obtained in approximate non-perturbative approaches [2, 3]. Recently some attempts have been made to determine the magnetic screening mass through the analysis of gap equations 四, 5, 6.

It is the purpose of this paper to discuss a detailed study of the temperature dependence of the magnetic screening mass, $m_{m}(T)$, obtained from calculations of the finite temperature gluon propagator on the lattice. Early lattice studies have attempted to extract this quantity from gauge invariant observables, using lattices with twisted boundary conditions [7, 8]. Likewise the electric screening mass usually is extracted from gauge invariant Polyakov loop correlation functions. Here we will determine the effective gluon masses directly from the long-distance behaviour of the gluon propagator. Although this requires the fixing of a gauge and leads to a gauge dependent definition of a screening mass, it has the advantage that it is closest to the perturbative definition of these masses and allows a direct comparison with perturbative calculations. A more detailed analysis of the momentum space representation of the gluon propagator may, however, also lead to the determination of a gauge independent pole mass [9].

We have performed calculations on large lattices of size $N_{\sigma}^{3} \times N_{\tau}$. In most cases we use $N_{\tau}=8$, which insures that the calculations in the high temperature phase are performed at gauge couplings well inside the scaling region of the $S U(2)$ lattice gauge theory, i.e. $\beta \geq 2.6$. For the spatial lattice we generally use $N_{\sigma}=32$, which allows us to analyze correlation functions up to distances $z T=2$. We have performed calculations in a large temperature interval from $T \simeq 1.3 T_{c}$ up to $T \simeq 16 T_{c}$ in order to become sensitive to possible logarithmic corrections to the leading linear dependence of the high temperature screening masses on $T$. Typically we have generated 800 independent gauge field configurations on which we fix the Landau gauge, $\left|\partial_{\mu} A^{\mu}(\mathbf{x})\right|^{2}=0$, in order to analyze gluon correlation functions. For the gauge fixing we use a combination of overrelaxation and FFT algorithms [10, 11]. 
We have discussed the performance of this algorithm in Ref. [12].

We define the gauge fields, $A^{\mu}(\mathbf{x})$, in the usual way from the $S U(2)$ link variables, $U_{\mu}(\mathbf{x})$, the dynamical variables in the lattice formulation,

$$
A_{\mu}(\mathbf{x})=\frac{-i}{2 g}\left(U_{\mu}(\mathbf{x})-U_{\mu}^{\dagger}(\mathbf{x})\right) .
$$

In a perturbative context the electric and magnetic screening masses are defined through the zero momentum limit of the gluon polarization tensor, $\Pi_{\mu \mu}\left(p_{0}, \vec{p}\right)$, at vanishing Matsubara frequency $p_{0}$. In coordinate space this limit can be realized through the long distance behaviour of correlation functions of gauge field operators in one of the spatial directions of the lattice, e.g. in the $z$-direction,

$$
G_{\mu}(z)=\left\langle\operatorname{Tr} A_{\mu}(z) A_{\mu}(0)\right\rangle,
$$

where the fields $A_{\mu}(z)=\sum_{x_{0}, x_{1}, x_{2}} A_{\mu}\left(x_{0}, \vec{x}\right)$ are obtained by averaging over a hyperplane transverse to the $z$-direction. In this way we project onto $p_{0}=p_{1}=p_{2}=0$ and the limit $p_{3} \rightarrow 0$ corresponds to $z \rightarrow \infty$. The long-distance behaviour of $G_{e}(z) \equiv G_{0}(z) \sim \exp \left\{-\tilde{m}_{e} z\right\}$ is related to the electric screening mass, while $G_{m}(z) \equiv 0.5\left(G_{1}(z)+G_{2}(z)\right) \sim \exp \left\{-\tilde{m}_{m} z\right\}$ yields the magnetic mass $\rrbracket^{\natural}$. Due to the Landau gauge condition, it is easy to see that $G_{3}(z)=$ const. [13].

A problem which may arise with the fixing of the Landau gauge is the occurrence of the so-called Gribov ambiguity [14]. The Landau gauge condition $\left|\partial_{\mu} A^{\mu}(\mathbf{x})\right|^{2}=0$ is realized on each lattice configuration by maximizing the trace of the link fields, $U_{\mu}(x)$,

$$
\Sigma=\sum_{\mu, x} \operatorname{Tr}\left[U_{\mu}(x)+U_{\mu}^{\dagger}(x)\right],
$$

in the space of gauge equivalent fields [10, 11, 12]. This maximization procedure, however, is not unique. If one performs on an ungauged configuration first a random gauge transformation and then maximizes $\Sigma$, it is possible that one ends in a different

${ }^{a}$ We denote by $\tilde{m}$ masses in lattice units. As all calculations, except one, have been performed on lattices with $N_{\tau}=8$ sides in the temporal direction, the masses in units of the temperature are given by $m / T=N_{\tau} \tilde{m}=8 \tilde{m}$. 
Gribov sector. To investigate the dependence of $m_{e}(T)$ and $m_{m}(T)$ on Gribov copies, we took 100 configurations, created on each of them 25 random gauge copies, and then performed the gauge fixing. Indeed, we find different Gribov copies, which are distinguished by the value of $\Sigma$. In order to test the sensitivity of $m_{e}(T)$ and $m_{m}(T)$ on this we selected the two samples of 100 configurations with smallest and largest value of $\Sigma$, respectively. On each sample we calculated the screening masses from the exponential decay of the correlation functions $G_{e}$ and $G_{m}$. Within our statistical accuracy we do not see any dependence of the screening masses on the different Gribov sectors.
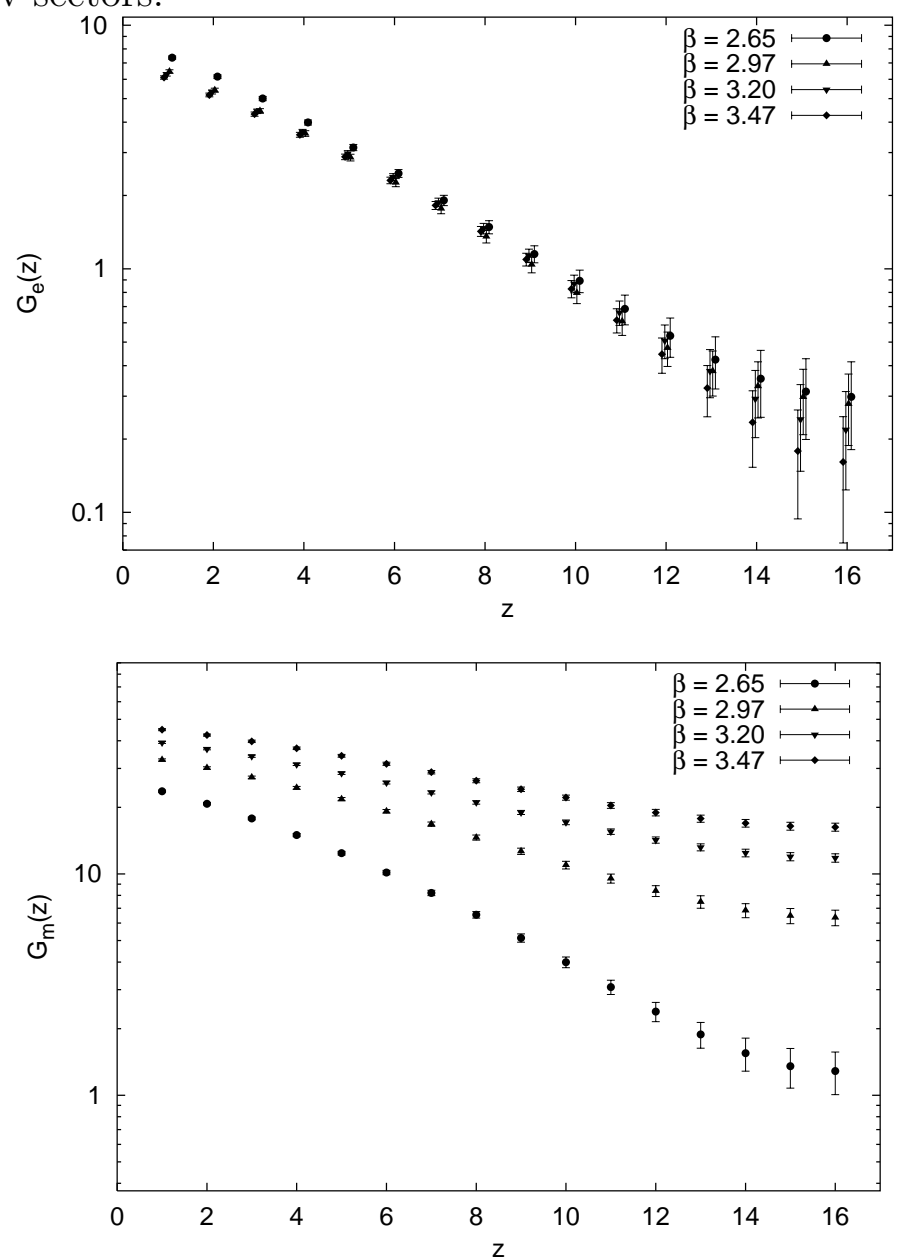

Figure 1: The electric, $G_{e}(z)$, and magnetic, $G_{m}(z)$, correlation functions for various values of the gauge coupling as a function of $z$ calculated on lattices of size $32^{3} \times 8$. The temperatures corresponding to the different $\beta$-values can be found in Table 1 . They cover the interval $T / T_{c} \in[1.5,16]$. The data points in the upper figure have been displaced horizontally for better viewing.

In Fig. 1 we show the electric and magnetic gluon propagators at various tem- 
peratures. Already from this figure it is obvious that the two correlation functions lead to quite different temperature dependences of $m_{m} / T=8 \tilde{m}_{m}$ and $m_{e} / T=8 \tilde{m}_{e}$. While $m_{e} / T$ does seem to be temperature independent, the magnetic mass clearly rises slower than linear in $T$, i.e. the correlation function $G_{m}(z)$ becomes flatter with increasing temperature, which suggests a decrease of $m_{m} / T$.

We have analyzed the long-distance behaviour of the correlation functions by studying the behaviour of local masses,

$$
\frac{G_{i}(z)}{G_{i}(z+1)}=\frac{\cosh \left(\tilde{m}_{i}\left(z-\frac{N_{\sigma}}{2}\right)\right)}{\cosh \left(\tilde{m}_{i}\left(z+1-\frac{N_{\sigma}}{2}\right)\right)} \quad, \quad i=e, m
$$

as well as by fitting the correlation functions with the ansatz, $G_{i}(z)=c \cosh \left(\tilde{m}_{i}(z-\right.$ $\left.N_{\sigma} / 2\right)$ ). From an analysis of the local masses we find that they slowly approach a plateau for $z T>1$. This behaviour is shown in Fig. 2 for $\beta=3.12$.

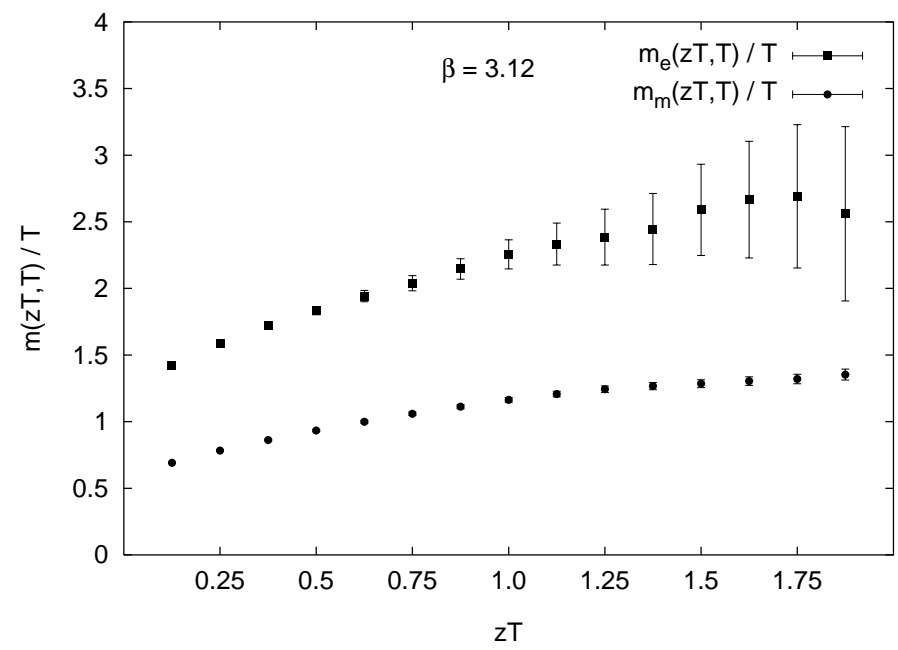

Figure 2: The local electric and magnetic screening masses for $\beta=3.12$ in the range $z T \in[0.125,1.875]$, i.e. $1 \leq z \leq 15$.

Also the single-cosh fits yield acceptable $\chi^{2}$-values only, if the short distance part of the correlation functions $(z T<1)$ is left out from the fit. This is obvious from Fig. 1. We generally observe that a fit to the propagator for $z T>1$ yields masses which are consistent within statistical errors with the local masses extracted at $z T=1(z=8)$. In Table 1 we give the results from our fits at all values of the gauge coupling analyzed. These results are also shown in Fig. 3. In order to 


\begin{tabular}{|l|r||l|l|}
\hline \multicolumn{1}{|c|}{$\beta$} & $T / T_{c}$ & $\frac{m_{e}(T)}{T}$ & $\frac{m_{m}(T)}{T}$ \\
\hline \hline 2.60 & 1.32 & $2.28(15)$ & $2.42(10)$ \\
\hline $2.74^{(1)}$ & 1.33 & $2.83(27)$ & $1.91(12)$ \\
\hline 2.65 & 1.53 & $2.25(17)$ & $2.25(9)$ \\
\hline $2.74^{(2)}$ & 2.00 & $2.69(10)$ & $1.92(3)$ \\
\hline 2.88 & 3.03 & $2.36(14)$ & $1.63(5)$ \\
\hline 2.97 & 3.93 & $2.26(15)$ & $1.46(5)$ \\
\hline 3.12 & 6.01 & $2.90(22)$ & $1.24(4)$ \\
\hline 3.20 & 7.53 & $2.40(15)$ & $1.17(3)$ \\
\hline 3.34 & 11.10 & $2.26(14)$ & $1.10(4)$ \\
\hline 3.47 & 15.88 & $2.57(15)$ & $1.06(4)$ \\
\hline
\end{tabular}

Table 1: Electric and magnetic screening masses in units of the temperature, $m_{e}(T) / T$ and $m_{m}(T) / T$ for temperatures $1.32 \leq T / T_{c} \leq 15.88$. Calculations have been performed on lattices of size $32^{3} \times 8$ except for the cases (1) and (2), where calculations have been performed on a $32^{3} \times 12$ (1) and a $32^{2} \times 64 \times 8$ (2) lattice. The masses have been obtained from fits to the correlation functions for distances $z T \geq 1$.

relate the gauge coupling used in the calculation to a temperature we make use of a parameterization of asymptotic scaling violations of the $S U(2) \quad \beta$-function [15].

We clearly see that the electric screening mass is proportional to the temperature. Up to temperatures of $16 T_{c}$ we do not see any indication for the expected perturbative behaviour $m_{e} \sim g(T) T$. Averaging over the results for $T \geq 2 T_{c}$ we obtain

$$
m_{e}(T)=(2.484 \pm 0.052) T .
$$

This agrees with the findings of a recent analysis of Polyakov loop correlation functions, where the electric mass has been determined with the help of the transfer matrix approach [16]. We will return to a more detailed discussion of the electric mass after having analyzed the magnetic screening mass.

The magnetic screening mass clearly rises slower than proportional to $T$. We thus may compare the results with the expected behaviour $m_{m} \sim g^{2}(T) T$. In Fig. ?? 


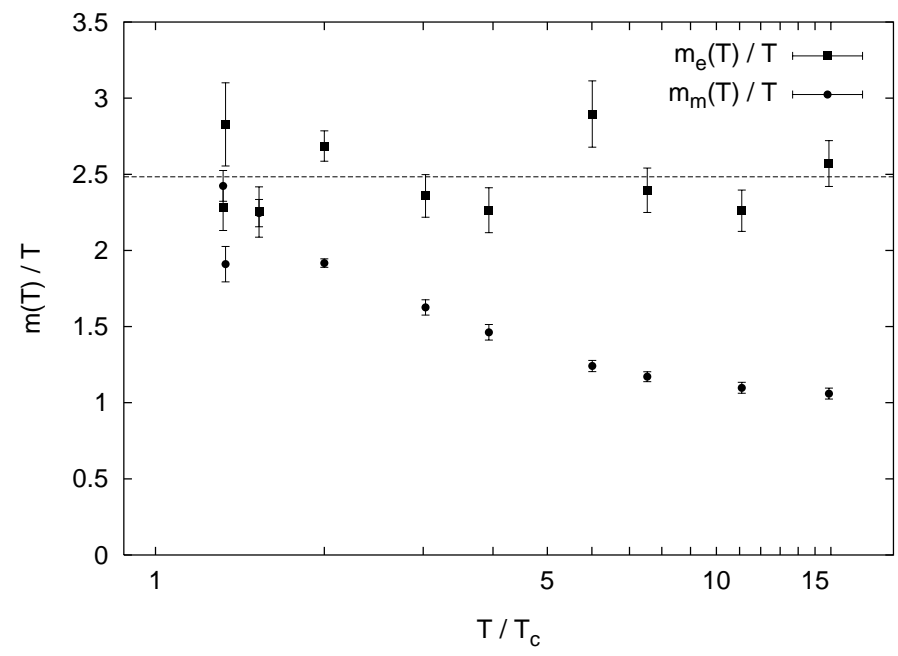

Figure 3: Electric and magnetic screening masses in units of the temperature versus $T / T_{c}$. The screening masses have been extracted from fits to the gluon correlation functions for distances $z T \geq 1$. The dashed line shows the fit to the electric mass given in Eq.(5)

we show the inverse mass, $T / m_{m}(T)$. This should rise logarithmically, if the gauge coupling is running according to the leading orders of the renormalization group equation, $g^{-2}(T) \sim \ln \left(T / T_{c}\right)$. We note that this does indeed describe our numerical results quite well. The spatial string tension is also expected to be sensitive to magnetic screening in the high temperature phase and indeed a similar scaling behaviour has been observed for it in [17].

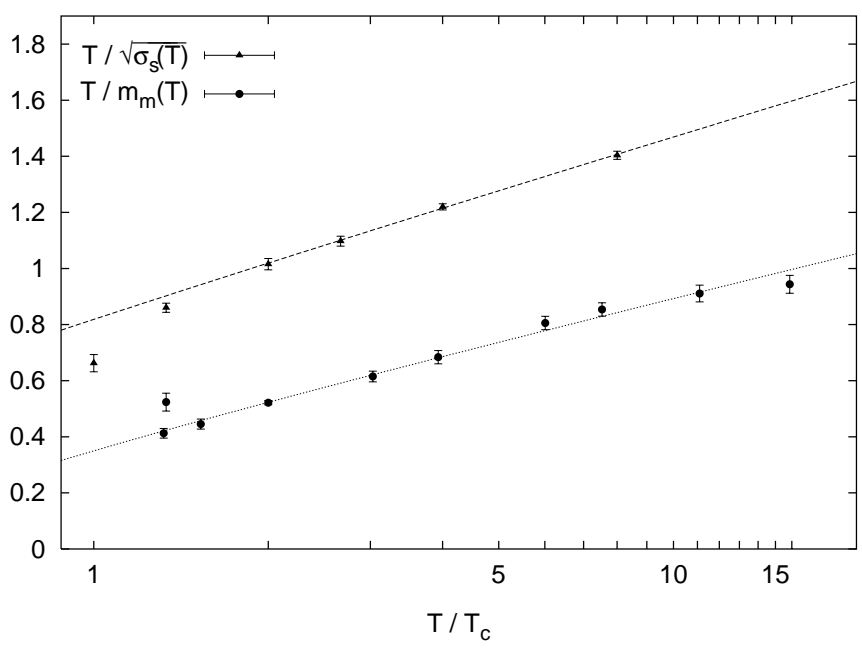

Figure 4: Inverse magnetic mass and square root of the spatial string tension (from Ref. [17]) versus $T / T_{c}$. The lines show the fits given in Eqs.(8) and (9). 
We have fitted the magnetic mass with the ansatz

$$
\frac{T}{m_{m}(T)}=c g^{-2}(T)
$$

assuming the validity of the two-loop formula for $g^{2}(T)$ with a free $\Lambda$ parameter,

$$
g^{-2}(T)=\frac{11}{12 \pi^{2}} \ln T / \Lambda_{m}+\frac{17}{44 \pi^{2}} \ln \left(2 \ln T / \Lambda_{m}\right)
$$

From a fit of the numerical results for $T \geq 2 T_{c}$ we find

$$
m_{m}(T)=(0.466 \pm 0.015) g^{2}(T) T
$$

with $\Lambda_{m}=0.262(18) T_{c}$. We note that our result for the magnetic mass is about twice as large as the earlier numerical results [7, 8]. This may not be too surprising in view of the rather small lattices used in these first studies larger than analytic results based on a semiclassical approximation [18] and gap equations [5, 6].

It is interesting to relate the magnetic mass to the spatial string tension, which also has been found to scale like $g^{2} T$ [17]. The temperature dependent running coupling has been found to be somewhat smaller in the case of $\sqrt{\sigma_{s}}$, which results from the different constant terms that went into the definition of the $\Lambda$-parameter. We may, however, express the result for the spatial string tension in terms of the coupling $g^{2}(T)$ determined here from the scaling of the magnetic mass. This yields

$$
\sqrt{\sigma_{s}}=(0.368 \pm 0.017)\left\{1-(0.113 \pm 0.022) g^{2}(T)\right\} g^{2}(T) T
$$

Combining this with Eq. (8) we find

$$
m_{m}(T)=(1.27 \pm 0.10)\left\{1+(0.113 \pm 0.022) g^{2}(T)\right\} \sqrt{\sigma_{s}}
$$

The spatial string tension has also been found to be quantitatively closely related to the string tension, $\sqrt{\sigma_{3}}$, of the three-dimensional $S U(2)$ gauge theory. We thus can

\footnotetext{
${ }^{\mathrm{b}}$ The analysis performed in Ref. [7] could only probe distances $z T \leq 0.5$ and the analysis in Ref. [8] has been performed at a value of the gauge coupling which was too large to keep the system confined in spatial directions.
} 
use the above relation to compare the result for the magnetic mass of the $(3+1)$ dimensional $S U(2)$ gauge theory at finite temperature with the mass gap of the three-dimensional $S U(2)$ theory. The latter is found to be about $5 \sqrt{\sigma_{3}}$ [19], i.e. (4-5)-times larger than the magnetic mass found here. It will be interesting to also analyze, in the future, the effective gluon mass in the three-dimensional gauge theory within the approach discussed here. This will clarify whether the gluon masses can be interpreted as constituent masses of the glueballs and to what extend the thermal gluon mass in $(3+1)$ dimensions and the gluon mass in three dimensions are related in a similar way as $\sigma_{s}(T)$ and $\sigma_{3}$.

In view of the discussion of the magnetic mass we shall now return to the result for the electric mass given in Eq. (5). The running coupling extracted from the magnetic mass varies by a factor two in the temperature interval studied by us, $g^{2}\left(2 T_{c}\right) \simeq 4.1$ and $g^{2}\left(16 T_{c}\right) \simeq 2.2\left(\right.$ similar values have been obtained from $\left.\sqrt{\sigma_{s}}\right)$. From the leading order perturbative result, $m_{e}(T)=\sqrt{2 / 3} g(T) T$, we thus would expect a $30 \%$ drop of $m_{e} / T$ in this temperature interval. Moreover, using the perturbative form at our largest temperature, we would conclude that the coefficient in front of $g(T) T$ comes out to be a factor two larger than suggested by leading order perturbation theory. Such an enhancement is also found when higher order effects, including the contribution from a non-vanishing mass, are taken into account through resumed perturbation theory [9]. It thus seems that indeed non-perturbative effects give large contributions to the electric screening mass and eliminate the leading $g(T)$-dependence. It will be interesting to perform calculations at even higher temperatures in order to see whether contact with leading order perturbative behaviour can be made at all in the electric sector. Work in this direction is in progress.

Acknowledgements: The work of UMH was supported in part by the DOE under grants \# DE-FG05-85ER250000 and \# DE-FG05-92ER40742 and the work of FK in part by the NATO research grant CRG940451. The computations have been performed on Connection Machines at the HLRZ, and SCRI. We thank the staff of these institutes for their support.

\section{References}


[1] A.D. Linde, Phys. Lett. 96B (1980) 289.

[2] K. Kajantie and J. Kapusta, Phys. Lett. 110B (1982) 299.

[3] O.K. Kalashnikov, Phys. Lett. B279 (1992) 367.

[4] J.R. Espinosa, M. Quirós and F. Zwirner, Phys. Lett. B314 (1993) 206.

[5] W. Buchmüller, Z. Fodor, T. Helbig and D. Walliser, Ann. Phys. 234 (1994) 260.

[6] O. Philipsen, On the Problem of the Magnetic Mass, to appear in the Proc. NATO Advanced Research Workshop on Electroweak Physics and the Early Universe, March 1994, Sintra, Portugal.

[7] A. Billoire, G. Lazarides and Q. Shafi, Phys. Lett. 103B (1981) 450.

[8] T.A. DeGrand and D. Toussaint, Phys. Rev. D25 (1982) 526.

[9] A.K. Rebhan, Phys. Rev. D48 (1993) R3967 and Nucl. Phys. B430 (1994) 319.

[10] C.T.H. Davies, G.G. Batrouni, G.R. Katz, A.S. Kronfeld, G.P. Lepage, K.G. Wilson, P. Rossi and B. Svetitsky, Phys. Rev. D37 (1988) 1581.

[11] J.E. Mandula and M. Ogilvie, Phys. Lett. B248 (1990) 156.

[12] F. Karsch and J. Rank, Nucl. Phys. B (Proc. Suppl.) 42 (1995) 508.

[13] J.E. Mandula and M. Ogilvie, Phys. Lett. 201B (1988) 117.

[14] V.N. Gribov, Nucl. Phys. B139 (1978) 1.

[15] J. Engels, F. Karsch and K. Redlich, Nucl. Phys. B435 (1995) 295.

[16] J. Engels, V.K. Mitrjushkin and T. Neuhaus, hep-lat/9412003 (to be published in Nucl. Phys. B).

[17] G.S. Bali, J. Fingberg, U.M. Heller, F. Karsch and K. Schilling, Phys. Rev. Lett. 71 (1993) 3059.

[18] T.S. Biró and B. Müller, Nucl. Phys. A561 (1993) 477.

[19] M. Teper, Phys. Lett. 289B (1992) 115. 